\title{
ङु \\ Time-resolved imaging of domain pattern destruction and recovery via nonequilibrium magnetization states
}

\author{
Philipp Wessels, ${ }^{1,2, *}$ Johannes Ewald, ${ }^{3}$ Marek Wieland, ${ }^{1,2}$ Thomas Nisius, ${ }^{3}$ Andreas Vogel,${ }^{4}$ Jens Viefhaus, ${ }^{5}$ \\ Guido Meier, ${ }^{1,4,6}$ Thomas Wilhein, ${ }^{3}$ and Markus Drescher ${ }^{1,2}$ \\ ${ }^{1}$ The Hamburg Centre for Ultrafast Imaging (CUI), Luruper Chaussee 149, 22761 Hamburg, Germany \\ ${ }^{2}$ Institut für Experimentalphysik, University of Hamburg, Luruper Chaussee 149, 22761 Hamburg, Germany \\ ${ }^{3}$ Institute for X-Optics, University of Applied Sciences Koblenz, RheinAhrCampus, Joseph-Rovan-Allee 2, 53424 Remagen, Germany \\ ${ }^{4}$ Institut für Angewandte Physik, University of Hamburg, Jungiusstraße 11, 20355 Hamburg, Germany \\ ${ }^{5}$ Deutsches Elektronen-Synchrotron (DESY), Notkestraße 85, 22607 Hamburg, Germany \\ ${ }^{6}$ Max Planck Institute for the Structure and Dynamics of Matter, Luruper Chaussee 149, 22761 Hamburg, Germany
}

(Received 3 September 2014; revised manuscript received 23 October 2014; published 17 November 2014)

\begin{abstract}
The destruction and formation of equilibrium multidomain patterns in permalloy $\left(\mathrm{Ni}_{80} \mathrm{Fe}_{20}\right)$ microsquares has been captured using pump-probe $\mathrm{x}$-ray magnetic circular dichroism (XMCD) spectromicroscopy at a new full-field magnetic transmission soft $\mathrm{x}$-ray microscopy endstation with subnanosecond time resolution. The movie sequences show the dynamic magnetization response to intense Oersted field pulses of approximately 200-ps root mean square (rms) duration and the magnetization reorganization to the ground-state domain configuration. The measurements display how a vortex flux-closure magnetization distribution emerges out of a nonequilibrium uniform single-domain state. During the destruction of the initial vortex pattern, we have traced the motion of the central vortex core that is ejected out of the microsquare at high velocities exceeding $1 \mathrm{~km} / \mathrm{s}$. A reproducible recovery into a defined final vortex state with stable chirality and polarity could be achieved. Using an additional external bias field, the transient reversal of the square magnetization direction could be monitored and consistently reproduced by micromagnetic simulations.
\end{abstract}

DOI: 10.1103/PhysRevB.90.184417

PACS number(s): 75.78.Fg, 78.47.da, 75.70.Kw, 68.37.Yz

\section{INTRODUCTION}

Most of modern data storage technology is based on the local manipulation of the magnetization in thin films or micro- and nanoelements. A fundamental investigation of the destruction and formation of domains on their natural length and time scales may contribute to improve the performance of nonvolatile magnetic storage devices as well as spintronic logic devices and meet the demands toward higher data densities and faster access times. Ideally, a direct time-resolved detection of the magnetization in real space with sufficient spatial and temporal resolution in form of a movie sequence that captures the evolution and reorganization of domains in magnetic materials is desirable. Essential processes like the emergence of an equilibrium multidomain pattern out of a single domain state have not been imaged directly in the time-domain.

For this purpose, we use a new time-resolving full-field soft $\mathrm{x}$-ray microscopy endstation to image the magnetization dynamics of small structures with sub-100-nm spatial and subnanosecond temporal resolution. Our setup features short and intense magnetic Oersted field pulses strong enough to destroy the ground state multidomain pattern in permalloy $\left(\mathrm{Ni}_{80} \mathrm{Fe}_{20}\right)$ squares. This enables the first direct observation of domain destruction and recovery out of a highly excited unstable magnetization state at these time scales.

Magnetic permalloy squares feature a Landau flux-closure magnetization pattern in the ground state with a vortex core in the center [1-3]. Such an element represents a two-bit memory cell depending on the sense of rotation of the in-plane magnetization (clockwise or counter-clockwise)

\footnotetext{
*philipp.wessels@desy.de; http://www.cui.uni-hamburg.de/
}

called chirality $c= \pm 1$ and the out-of-plane alignment of the vortex core (up or down) called polarity $p= \pm 1$. Advanced knowledge of the domain configuration manipulation in these samples is of great importance for the development of multibit vortex data storage devices [4,5].

Previous research was mainly focused on the dynamics initiated by small deflections of the vortex core from the center using spin currents [6-9] or dynamic external fields of typically a few millitesla amplitude [10-15]. The result of this excitation is an oscillation around the center in form of a gyrotropic eigenmode constrained by a parabolic potential [16]. Stronger stimulation of the core leads to switching of the polarity and the emission of spin-waves $[17,18]$ if the vortex core exceeds a critical velocity around $\approx 300 \mathrm{~m} / \mathrm{s}$ [19-21]. This work addresses the question what happens if the vortex core is accelerated by intense field pulses with fast slopes so that it can not perform a complete round trip until it is ejected. Moreover, the stability of vortex chirality and polarity after recovery are studied.

\section{EXPERIMENTAL SETUP}

The $2 \times 2-\mu m^{2}$ permalloy $\left(\mathrm{Ni}_{80} \mathrm{Fe}_{20}\right)$ squares of $30-\mathrm{nm}$ thickness are fabricated by electron-beam lithography, physical vapor deposition (PVD) and lift-off processing on a 200-nm thick silicon-nitride membrane. A 300-nm thick copper layer in form of a coplanar waveguide (CPW) is manufactured on top of the resulting sample by ultraviolet (UV) photolithography, PVD and lift-off processing. The waveguide layer is protected against oxidation by a 5 -nm gold cap layer.

The experiments are carried out at the soft x-ray beamline P04 [22] of the high-brilliance third generation synchrotron 
radiation source PETRA III at DESY in Hamburg. The undulator beamline provides a high flux of monochromatized circular polarized photons in the energy range of 250 to $3000 \mathrm{eV}$ with variable helicity ideal for $\mathrm{x}$-ray magnetic circular dichroism (XMCD) measurements [23]. In the operation mode for timeresolved experiments, the PETRA III storage ring is filled with 40 bunches circulating at a revolution frequency of $130.1 \mathrm{kHz}$ so that the P04 beamline delivers a soft $\mathrm{x}$-ray flash of 38-ps root mean square (rms) duration [24] every $192 \mathrm{~ns}$.

We have set up a new time-resolving full-field magnetic transmission X-ray microscope (TR-MTXM) [25-27] that can be attached to the beamline. A grating condenser [28] consisting of 940 diffraction gratings of $25 \times 25-\mu \mathrm{m}^{2}$ size and an included central stop homogeneously illuminates the sample with a flat-top profile of $20-\mu \mathrm{m}$ diameter. The sample plane is directly imaged with a magnification of 700 onto a two-dimensional pixel detector by a micro zone plate of $80-\mu \mathrm{m}$ diameter and an outermost zone width of $50 \mathrm{~nm}$ limiting the spatial resolution of the instrument to $65 \mathrm{~nm}$ [27] according to the Rayleigh criterion. The numerical apertures of condenser and zone plate are matched and the geometry of the setup is depicted in Fig. 1. For the XMCD measurements, the instrument is operated at the nickel $L_{3}$ edge at a photon energy of $852.7 \mathrm{eV}$ with a bandpass of $1 \mathrm{eV}$. To access the in-plane magnetization component, the sample plane normal is tilted by $20^{\circ}$ with respect to the photon beam direction.

In order to excite the magnetic structures, short current pulses of $\tau=186 \mathrm{ps}$ rms duration synchronized with a residual jitter $<130$ ps rms to the soft x-ray pulses are applied to the coplanar waveguide. The inner conductor stripline has a width of $w=10 \mu \mathrm{m}$ resulting in an accompanied Oersted field of $\mu_{0} h_{0}=\mu_{0} I_{0} /(2 w)=82 \mathrm{mT}$ peak amplitude at $I_{0}=1.3 \mathrm{~A}$ peak current. This curled field surrounding the conductor line manipulates the magnetization of the permalloy layers in the vicinity of the waveguide. In order to keep the heat dissipated into the sample at a tolerable level, the current pulse period was kept at the $7.7 \mu$ s revolution period of the storage ring which corresponds to a duty cycle of $186 \mathrm{ps} / 7.7 \mu \mathrm{s}=2.4 \times 10^{-5}$. Thus only an x-ray microscope image generated by one of the 40 bunches in the storage ring contains dynamic information. The remaining 39 pulses have to be blocked to avoid a large background overlaying the time-resolved signal from the bunch directly following the pump pulse. The excessive pulses are filtered by a custombuilt fast gateable $\mathrm{x}$-ray detector [26,27]. Briefly, the $\mathrm{x}$-ray microscope image is projected onto a $\mathrm{P} 46$ phosphor screen that converts it into the visible spectral range. The emitted photons are transferred by a relay optic consisting of two fast camera lenses onto the photocathode of a commercial image intensifier tube. The intensifier features a single microchannel plate (MCP) amplification stage and another P46 phosphor output screen which is fiber-coupled to a cooled, back-illuminated charge-coupled device (CCD) camera. By applying a positive bias voltage to the photocathode, the $\mathrm{X}$-ray microscope image is blocked by default. A 30-ns long gate pulse of negative voltage opens the detector and transfers the image to the CCD chip and allows selecting the information contained in one particular bunch because two synchrotron light pulses are separated by 192 ns in 40 bunch mode. A suppression of the background with respect to the signal of the selected bunch exceeding one order of magnitude is possible [27]. A complete separation of the signal from adjacent bunches is not possible due to the afterglow of the P46 phosphor screen [29].

To capture the magnetization dynamics, XMCD images have been taken for several delay settings between pump and probe pulse. For a fixed delay, the dynamic signal of the pumped bunch is collected and integrated on the CCD chip of the camera. Here, the delay $\Delta t=0 \mathrm{~ns}$ corresponds to the image probing the sample exposed to the peak intensity of the Oersted field pulse (see below).

\section{RESULTS AND DISCUSSION}

The image sequence of Fig. 2 shows a movie (see Ref. [30]) of the magnetization dynamics in two $2 \times 2-\mu \mathrm{m}^{2}$ permalloy squares located between the conductor of the waveguide and the silicon nitride substrate. The width of the conductor ranges from the left to the right image border of one frame as indicated by the dashed orange line in Fig. 2. The XMCD map of one pump-probe delay setting is created by subtracting the counts

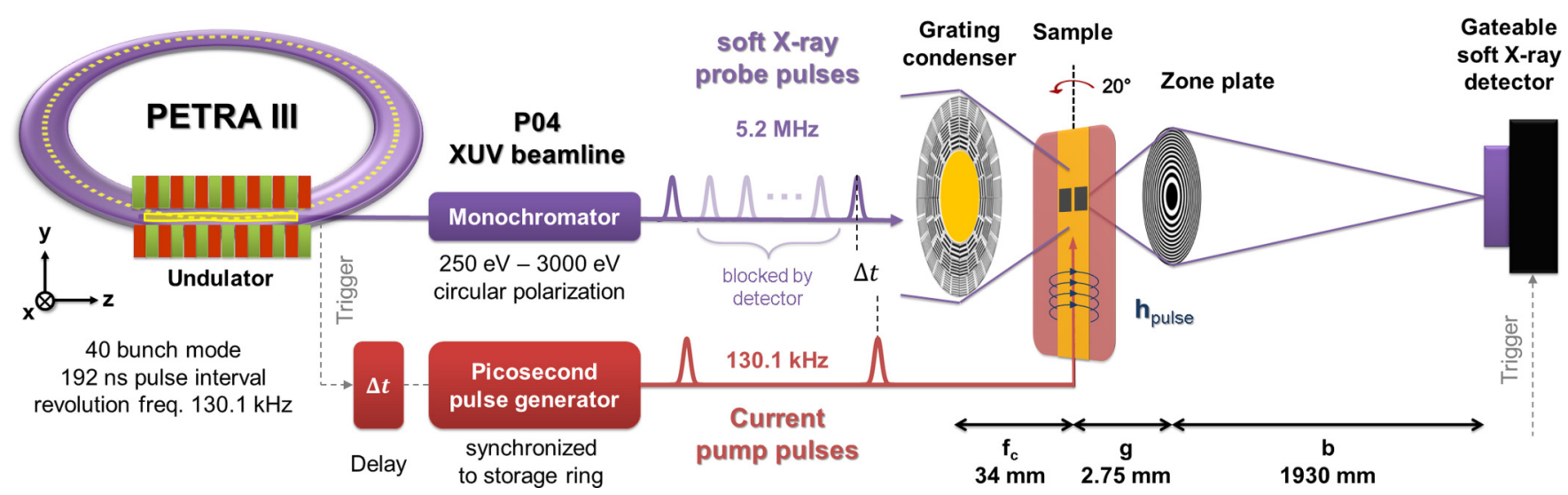

FIG. 1. (Color online) Time-resolving full-field magnetic transmission x-ray microscopy (TR-MTXM) setup. The grating condenser illuminates a $20-\mu \mathrm{m}$ diameter spot on the sample that is imaged by the micro zone plate with an outermost zone width of $50 \mathrm{~nm}$ onto a gateable 2D detector. The sample structures can be excited by short magnetic field pulses $\mathbf{h}_{\text {pulse }}$ formed by a transient current through a coplanar waveguide. 


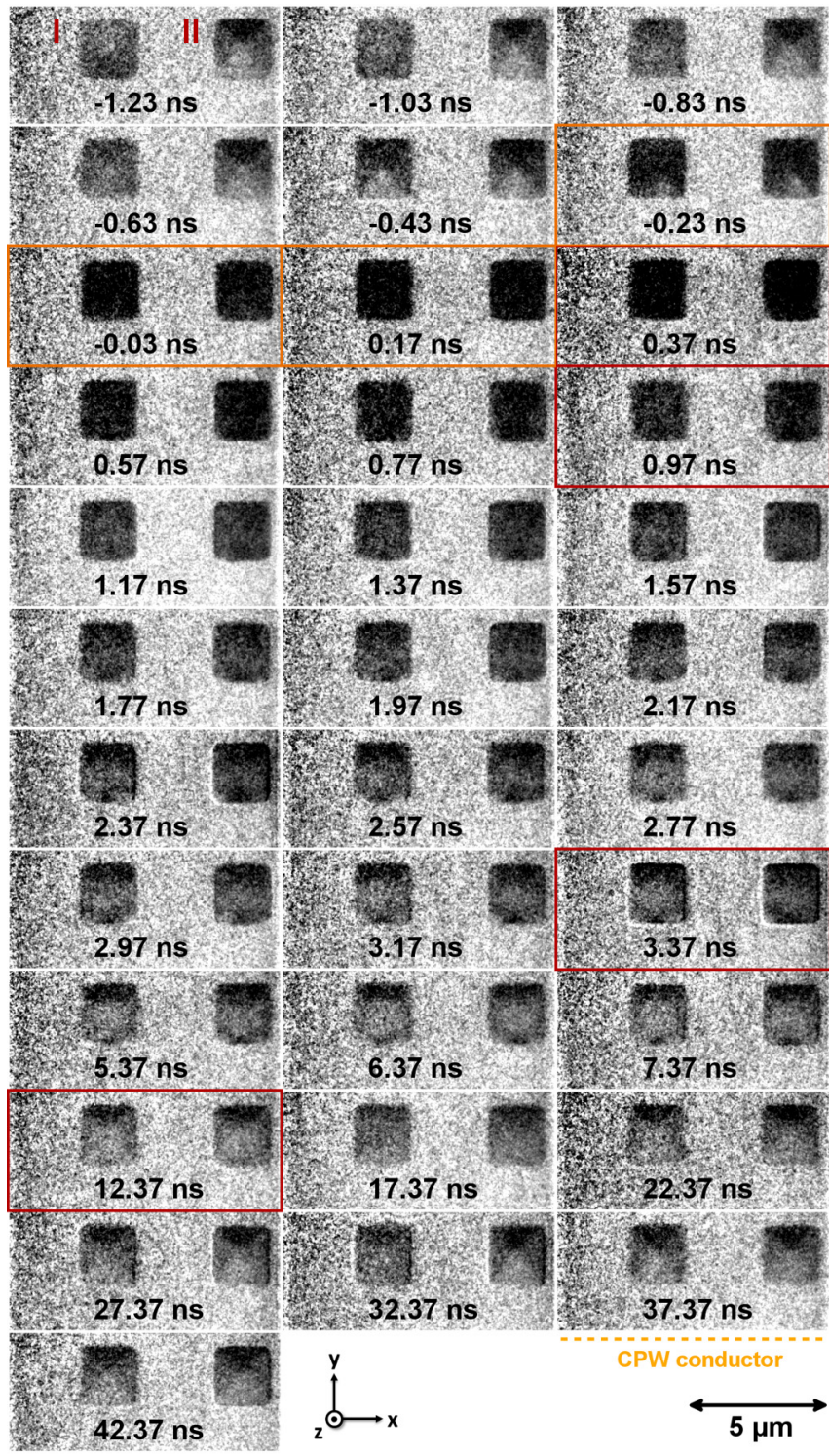

FIG. 2. (Color online) Image sequence of the magnetization dynamics of two $2 \times 2-\mu \mathrm{m}^{2}$ permalloy squares (I + II) exposed to a short magnetic field pulse of $\tau=186 \mathrm{ps}$ duration and $\mu_{0} h_{0} \approx 82 \mathrm{mT}$ peak amplitude at $\Delta t=0 \mathrm{~ns}$. The XMCD images recorded at different pump-probe delays $\Delta t$ show the Landau vortex domain pattern before the pulse, its destruction followed by a homogeneous magnetization during the pulse and the domain pattern recovery after the pulse has vanished. Each XMCD frame is generated by subtracting two images with 180-s exposure time at opposing photon helicities. Images within the full width at half maximum (FWHM) of the pulse are highlighted by orange frames and key images discussed in the text are marked by red frames.

of two images each exposed for $180 \mathrm{~s}$ at opposite photon helicities. Due to the tilted sample, the setup is sensitive to the magnetization $x$ and $z$ components which experience an overlap with the photon beam direction along the $z$ axis (see Fig. 1). The measured gray level linearly resembles the amplitude and direction of these components. The contribution of $M_{x}$ and $M_{z}$ can not be disentangled but due to the small thickness of the permalloy layer and the dominating shape

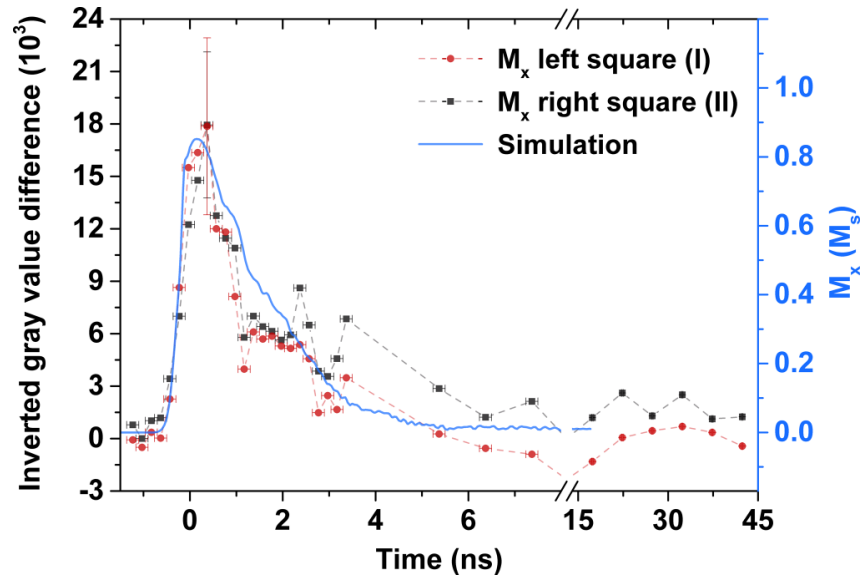

FIG. 3. (Color online) Spatially averaged inverted gray level of the permalloy squares I (red circles) and II (black squares) in the XMCD images of Fig. 2 corresponding to the total magnetization component $M_{x}$ with respect to time. Along with the data is plotted the $M_{x}$ output of a micromagnetic simulation (blue solid curve).

anisotropy [31], the $z$ component can be safely assumed to be negligible except in the vortex core itself. This means that the gray level maps the $M_{x}$ component of the magnetization where low (dark) values correspond to a positive alignment along the $x$ axis while high (bright) levels relate to negative $M_{x}$ amplitudes.

In the first frames, element number II shows a vortex magnetization pattern consisting of four domains before the pump pulse has arrived. During arrival of the Oersted field pulse indicated by orange frames in Fig. 2, the magnetization of the squares aligns along the pulsed field direction ( $x$ axis) leading to a measured dark contrast in the XMCD images. The following frames show the recovery of the domain structure back to the Landau flux-closure state.

Figure 3 traces the integrated gray level of both squares in the image sequence of Fig. 2 over time. For a fixed sample tilt angle and the available degree of circular photon polarization approaching unity at the P04 beamline, this signal is proportional to the spatially averaged $M_{x}$ amplitude. Both specimens show a similar dynamic behavior with a fast alignment along the pulsed Oersted field direction $\mathbf{h}_{\text {pulse }}(t)=h_{x}(t) \cdot \hat{\mathbf{x}}$ on a picosecond time scale and a slower decay on a nanosecond time scale to an equilibrated magnetization configuration where the number of domains pointing to positive and negative $x$ direction is evened out. The error bars represent the standard deviation of the gray values in the most aligned state where the majority of the magnetization is directed along the $x$ axis and serves as an upper limit for the uncertainty in the measurement. The actual error of the measurement is believed to be smaller because an inherent variation of the magnetization over the integrated area (i.e., domains pointing into different directions) also contributes to the standard deviation similar to a noise related variation. The agreement between the data points for the two squares (I + II) suggests that the error indeed is smaller than indicated by the standard deviation.

Before the arrival of the excitation pulse at the sample, the magnetization is evened out in the ground state of both elements. This corresponds to the apparent Landau pattern 
in element II where the vector sum of the magnetization is close to zero. Element number I shows a homogeneous gray shade possibly due to an unstable chirality of the final vortex state because the superposition of two vortex images with opposite chirality leads to a structureless gray XMCD image.

The rising edge of the signal is dominated by the duration of the Oersted field pulse convoluted with the synchronization jitter and shows a rapid slope within 300 ps (10\% to $90 \%)$. The transient magnetic field peaks at $\Delta t=0 \mathrm{~ns}$, however, the magnetization component $M_{x}$ reaches its maximum value 370 ps after the excitation pulse maximum. The energetically unfavorable uniform alignment of the magnetization corresponds to a large stray field and leads to a decay of the magnetization signal by the formation of domains due to the internal demagnetization field. This rearrangement happens on a pico- to nanosecond time scale and after $6 \mathrm{~ns}$ the $M_{x}$ signal has recovered to values comparable to the equilibrium configuration.

Figure 3 also displays the spatially averaged $M_{x}$ component in units of the saturation magnetization $M_{S}$ obtained by micromagnetic simulations carried out with the NMAG code [32,33]. The sample material is modeled by mapping the $2 \times 2 \times 0.03-\mu \mathrm{m}^{3}$ geometry and providing the saturation magnetization $\mu_{0} M_{S}=1.005 \mathrm{~T}$, exchange constant $A=13 \times 10^{-12} \mathrm{~J} / \mathrm{m}$ and damping constant $\alpha=0.01$ of a similarly processed permalloy layer [12]. For computational reasons, the maximum grid size in the simulation is set to $25 \mathrm{~nm}$ so that dynamics considering the vortex core are not accessible because the simulation cell volume is too large to capture the exchange energy in the vicinity of the vortex core. However, considering the remaining dynamics this approximation is reasonable for permalloy because of the dominating demagnetization energy compared to the exchange energy [34]. The excitation pulse is modeled by a Gaussian envelope

$$
h_{x}(t)=h_{0} \exp \left(-\frac{t^{2}}{2 \tau^{2}}\right)
$$

with a peak amplitude of $\mu_{0} h_{0}=88 \mathrm{mT}$ and a duration of $\tau=210 \mathrm{ps}$ corresponding to the experimental values of $82 \mathrm{mT}$ and $186 \mathrm{ps}$ (see above). The simulated magnetization response depends on peak amplitude and pulse duration and values differing more than $20 \%$ from the given experimental parameters do not reproduce our data set well any more. The slightly longer pulse duration necessary in the simulation compared to the measured pulse duration at the output port of the pulse generator is attributed to pulse broadening (dispersion and damping of frequency components) during transport to the magnetic system.

To calibrate the measured integral gray value signal to the magnetization output of the simulation, the $M_{x}$ axis of the simulation is vertically scaled to fit the span of the measured data points on the ordinate. Thus the integral gray value can be mapped to the magnetization in units of the saturation magnetization $M_{S}$. The time axis of the measured signal is calibrated by shifting the data points horizontally to a maximal overlap in the rising slope with the simulated signal. This determines the offset in the experimentally adjusted pump-probe delay settings. The temporal axis in the simulation is defined by the peak amplitude of the transient magnetic field pulse at $t=0 \mathrm{~ns}$.

A reasonable qualitative shape agreement is achieved for the rising edge and for the different decay slopes during reorganization to the equilibrium magnetization pattern. In the simulation, the most aligned state corresponds to $M_{x} \approx$ $0.85 M_{S}$ and is created $150 \mathrm{ps}$ after the excitation pulse reaches its peak amplitude at $t=0 \mathrm{~ns}$. The same simulation parameters also reproduce the measured magnetization decay for other permalloy sample geometries like circular disk structures [27] not shown here.

\section{A. Domain pattern destruction and vortex core motion}

The change of the magnetization according to the emerging field at the rising edge of the excitation pulse occurs fast on a time scale of a few hundred picoseconds. At $\Delta t=370 \mathrm{ps}$ (first red frame in Fig. 2), the magnetization is maximally aligned along the excitation field lines and features a homogeneous magnetization without any detectable substructure.

A closer look into the frames of Fig. 2 before the appearance of the uniform magnetization state reveals dynamic information on the vortex core motion during the destruction of the domain pattern. Tracing the position of the core frame by frame in Fig. 4 demonstrates that it is ejected on a curved trajectory to the bottom right edge. A slow adiabatic increase of the field amplitude would enlarge the domain pointing along the field lines until this domain dominates the sample element at the saturation magnetization $M_{S}$. In this case, the vortex core would leave the square on a straight trajectory. Here, the intense current pulse delivers high field gradients corresponding to a broad spectrum of frequency components. The response of the excited system is most efficient for modes with minimal losses and thus the observed trajectory curvature

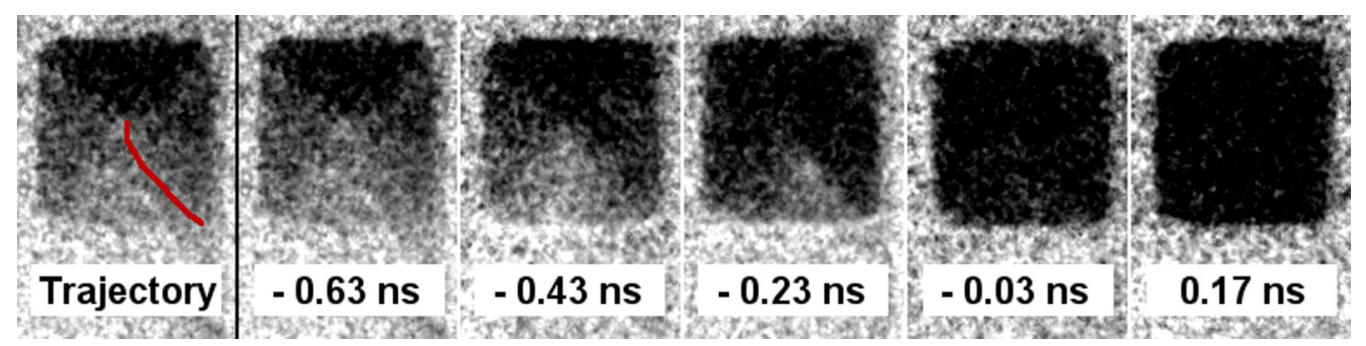

FIG. 4. (Color online) Trajectory of the vortex core motion in permalloy square II with the corresponding frames of Fig. 2 during the domain pattern destruction at the rising slope of the excitation pulse $\mathbf{h}_{\text {pulse }}$. 
TABLE I. Positions and velocities of the vortex core in square II of Fig. 4. The temporal uncertainty is dominated by the jitter of $130 \mathrm{ps}$ and the spatial inaccuracy by two pixel equivalent to $40 \mathrm{~nm}$ which is the closest value to the imaging resolution.

\begin{tabular}{lccccc}
\hline \hline & \multicolumn{2}{c}{ Position } & & \multicolumn{2}{c}{ Velocity } \\
\cline { 2 - 3 } \cline { 5 - 6 } Time $(\mathrm{ps})$ & $x(\mathrm{~nm})$ & $y(\mathrm{~nm})$ & & $v_{x}(\mathrm{~km} / \mathrm{s})$ & $-v_{y}(\mathrm{~km} / \mathrm{s})$ \\
\hline-630 & 0 & 158 & & $0.1 \pm 0.3$ & $1.1 \pm 1.0$ \\
-430 & 20 & -59 & & $0.4 \pm 0.2$ & $1.2 \pm 0.6$ \\
-230 & 178 & -317 & & $1.8 \pm 0.8$ & $2.1 \pm 1.0$ \\
-30 & 733 & -911 & & $1.7 \pm 0.8$ & $1.7 \pm 0.8$ \\
170 & 581 & -990 & & $0.6 \pm 0.6$ & $0.4 \pm 0.5$ \\
\hline \hline
\end{tabular}

originates from the excitation of the circular gyrotropic eigenmode of the vortex.

By further relating the positions of the vortex core to the time delay, it is possible to extract the velocities of the core motion collected in Table I. The position can be located with 40-nm (two pixel) accuracy according to the resolution of the soft $\mathrm{x}$-ray microscope and the temporal error is dominated by the synchronization jitter of $130 \mathrm{ps}$. The analysis yields unusually high velocities of up to $(2.1 \pm 1.0) \mathrm{km} / \mathrm{s}$ even when the rather large error interval of the velocity obtained by error propagation is considered.

Suppose the vortex core is driven into oscillation with its eigenfrequency of several $100 \mathrm{MHz}[10,12,16]$ by an alternating field. If now the oscillation amplitude of the core is gradually increased by resonant excitation or rising the alternating field amplitude, a reversal of the polarity is expected at core velocities exceeding $\approx 300 \mathrm{~m} / \mathrm{s}$ [19-21], which is not observed here. Due to the intense Oersted field amplitude and the fast rising edge of the excitation pulse, the magnetic vortex has not enough time to perform a complete round-trip on its eigenmode and is ejected before the motion is completed. Remarkably, the core motion can still be described by the movement of a rigid particle according to the Thiele model [35] and the validity of this model is now confirmed also for strong excitations and high velocities.

The fact that the trajectory is visible in the presented data acquired in a pump-probe experiment means that the motion is reproducible since a frame is created by averaging over approximately $2.3 \times 10^{7}$ pump-probe cycles at a constant delay setting. A reproducible final domain pattern and sense of rotation in the motion means that the vortex chirality as well as polarity remain constant. A recent work [36] claims that the Dzyaloshinskii-Moriya interaction with the sample substrate is responsible for the vortex state asymmetry favoring a certain configuration. Here, the shape of the excitation pulse together with sample imperfections are believed to be the dominating effect. Micromagnetic simulations using NMAG (see above) confirm that even without modeling the sample substrate the reproducible recovery is possible. Because the maximum magnetization alignment is below the saturation magnetization $\left[\max \left(M_{x}\right)<M_{S}\right.$ ], the information concerning chirality and polarity has to be contained in the residual magnetization vectors not pointing along the excitation pulse field direction $(x$ axis).

\section{B. Domain pattern recovery}

The recovery to the ground state domain pattern is traceable in the frames with a positive time stamp succeeding the excitation pulse in the image sequence of Fig. 2 where key images discussed in this section are highlighted by red frames. Both permalloy squares show similar recovery dynamics. Up to $1 \mathrm{~ns}$ the darkest gray value of the aligned state is homogeneously reduced without any noticeable substructure. This recovery period corresponds to the highest decay slope between 0.5 to $1 \mathrm{~ns}$ in the $M_{x}$ curves of Fig. 3 .

Subsequently, a substructure starts building up until $3.37 \mathrm{~ns}$ forming a zigzag domain aligned along $M_{x}$ (dark contrast) on the top and another small $M_{x}$ domain in the bottom center area, which is also prominent in the spatially resolved output of the NMAG simulation (see Ref. [37]). This section is attributed to the slower $M_{x}$ decay rate between 1 and 3 ns in Fig. 3.

At this stage, the magnetization has reached an arrangement with equal areas pointing to $M_{x}$ and in the opposite direction according to the vanishing $M_{x}$ amplitude in Fig. 3. However, the dynamics is not yet complete and it takes additionally several $10 \mathrm{~ns}$ to reorganize the transient magnetization state at $3.37 \mathrm{~ns}$ back to the final Landau state. During this reorganization phase $M_{x}$ remains at zero and the zigzag domains merge to a single vortex in the center while the small dark domain in the bottom is suppressed until it vanishes. In the last frames between 12 and 42 ns only the top $M_{x}$ domain grows until the vortex core is positioned in the center of the square structure.

A comparison with the spatially resolved NMAG output reveals that when the simulation approaches $M_{x}=0 M_{S}$ after $6 \mathrm{~ns}$, the computed magnetization pattern has almost reached the final Landau configuration. We attribute the longer reorganization phase to a balanced Landau pattern in the experimental data to sample imperfections and effects related to the exchange interaction, which is not exactly described in the simulation due to the unit cell size.

\section{Magnetization reversal in an external field}

The reaction of the magnetization to the short excitation pulses is also studied in an opposing external magnetic bias field and the resulting data of square I are presented in the image sequence of Fig. 5 (see Ref. [38] for a video). The frames were created by subtracting the counts of two images each exposed for $360 \mathrm{~s}$ taken at opposite photon helicities. The bias field generated between the poles of an iron core coil initially aligns the magnetization of the permalloy layer along $-x$ direction (white contrast).

When the pulse arrives, two domain walls are launched from the left and right side where the demagnetization field is maximal. As the transient field rises between -300 and $-100 \mathrm{ps,}$ the domains propagate toward the center, and at $-50 \mathrm{ps}$, the central area also reverses its magnetization direction from $-M_{x}$ to $M_{x}$. Around the maximum of the field amplitude, the square magnetization is aligned in $x$ direction (black contrast) and reverses to $-x$ after the pulse has vanished.

This measurement is also simulated with the NMAG code and the output frames of the magnetization vector field are presented in Fig. 6 (see Ref. [39] for a video). The gray value represents amplitude and orientation of the experimentally 


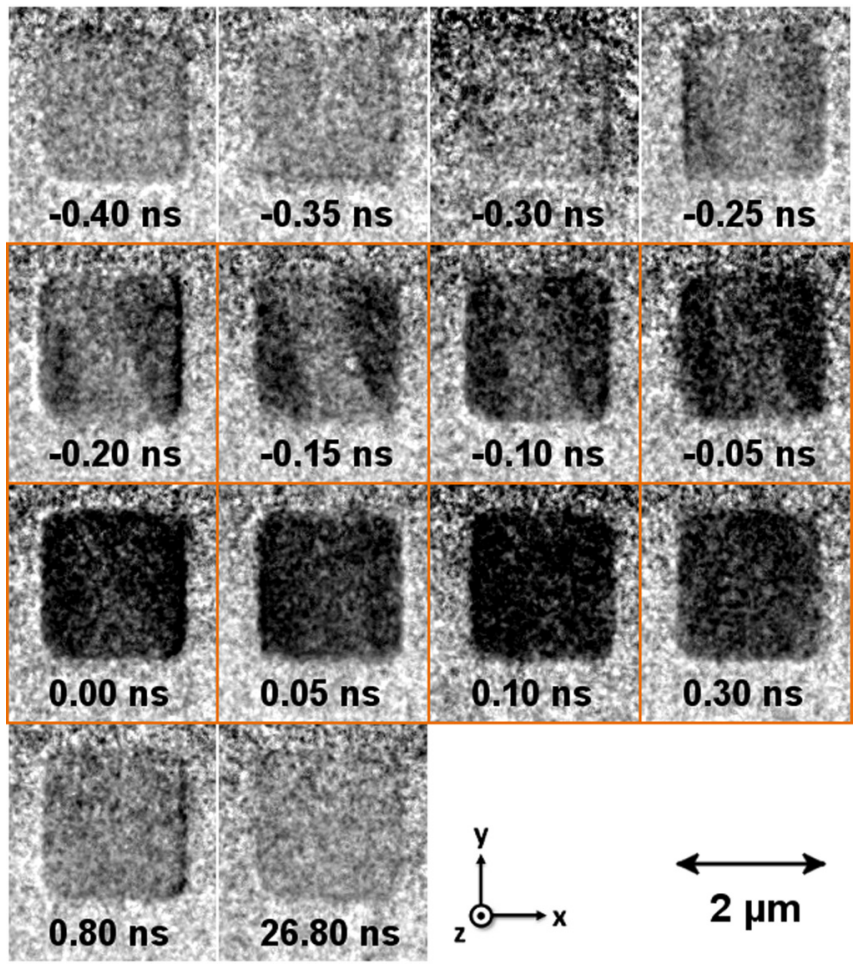

FIG. 5. (Color online) Magnetization reversal of the $2 \times 2-\mu \mathrm{m}^{2}$ permalloy square I of Fig. 2 in a static external magnetic field counteraligned to the transient excitation pulse field $\mathbf{h}_{\text {pulse }}$. Each $\mathrm{XMCD}$ frame is generated by subtracting two images exposed for $360 \mathrm{~s}$ at opposing photon helicities. Images within the pulse FWHM are highlighted by orange frames.

observable $M_{x}$ component (black: positive, white: negative) of the discretized magnetization vector $\mathbf{M}$. The sample geometry and material parameters as well as the excitation pulse are the same as used for the simulation generating the output in Fig. 3. Additionally, a constant external magnetic bias field of $\mu_{0} H_{\text {ext }}=38 \mathrm{mT}$ amplitude tilted at an angle of $5^{\circ}$ with respect to the $x$ axis that is counter-aligned to the transient field pulse direction is introduced. The tilt angle is added to avoid the academic situation that the torque between perpendicular field and magnetization vectors is zero. Furthermore, it accounts for inhomogeneities of the bias field lines and by varying the angle in the simulation the best result can be obtained for the here applied $5^{\circ}$ value. A remarkable agreement between the measurement in Fig. 5 and the simulation in Fig. 6 is achieved and the simulation is able to reproduce all features of the XMCD measurement frame by frame. Moreover, the simulation is consistent with the measurement in Fig. 2 where the same material parameters and pulse properties are applied. The simulation also contains two additional frames at 0.35 and $0.40 \mathrm{~ns}$ (red borders) during the reorganization to the initial state where no corresponding XMCD data are available.

Figure 7 depicts the inverse spatially averaged gray level of the XMCD frames in Fig. 5 along with the simulated total $M_{x}$ ratio in units of the saturation magnetization $M_{S}$. According to the simulation the magnetization is commonly aligned to $M_{x}=-0.9 M_{S}$ and twists to $M_{x}=+0.6 M_{S}$ during the subnanosecond excitation pulse. The rising slope in the

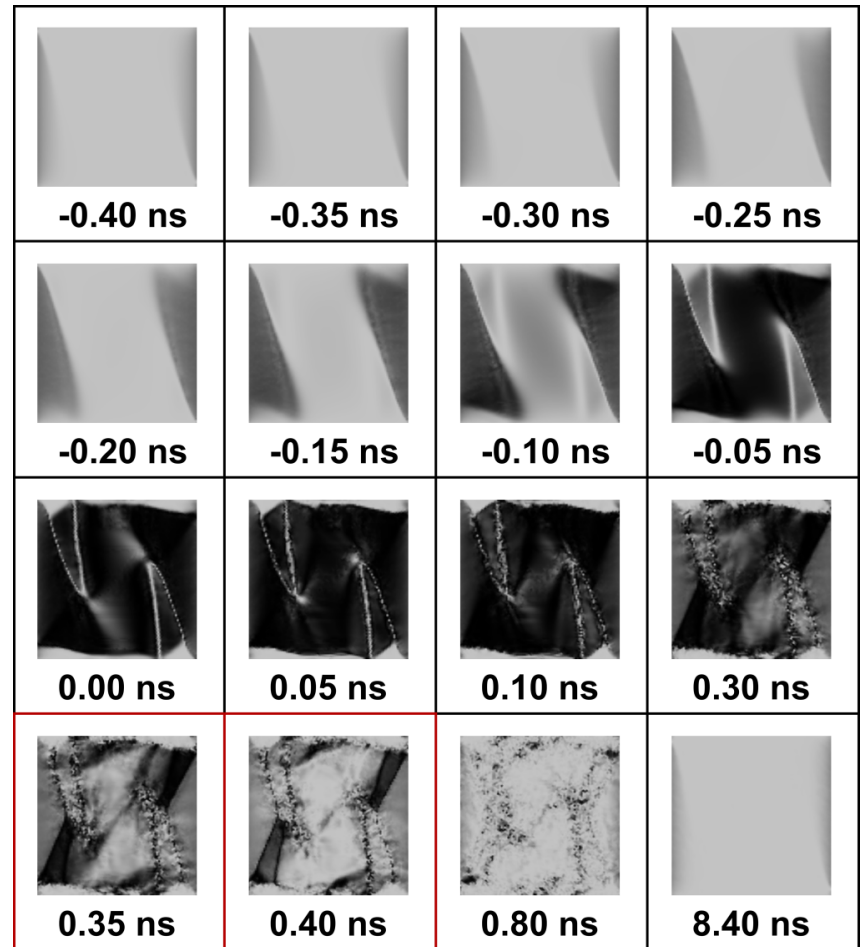

FIG. 6. (Color online) Simulated magnetization response of a $2 \times 2 \times 0.03-\mu \mathrm{m}^{3}$ permalloy square to the excitation pulse $\mathbf{h}_{\text {pulse }}$ of $\tau=210$ ps duration with an additional opposing external magnetic bias field of $\mu_{0} H_{\text {ext }}=38 \mathrm{mT}$ tilted by an angle of $5^{\circ}$ with respect to the $x$ axis. The output of the NMAG simulation displays the experimentally accessible $M_{x}$ component of discretized magnetization vectors M. Black indicates an orientation along the $x$ axis and white into the opposite direction. The red framed images at 0.35 and $0.40 \mathrm{~ns}$ also show the simulated decay back to the alignment along the bias field direction where no XMCD data are available.

simulation is higher compared to the measurement because the slope of the XMCD data points is smeared out by convolution with the PETRA III probe pulse duration of 38-ps rms [24] and timing jitter of 130-ps rms.

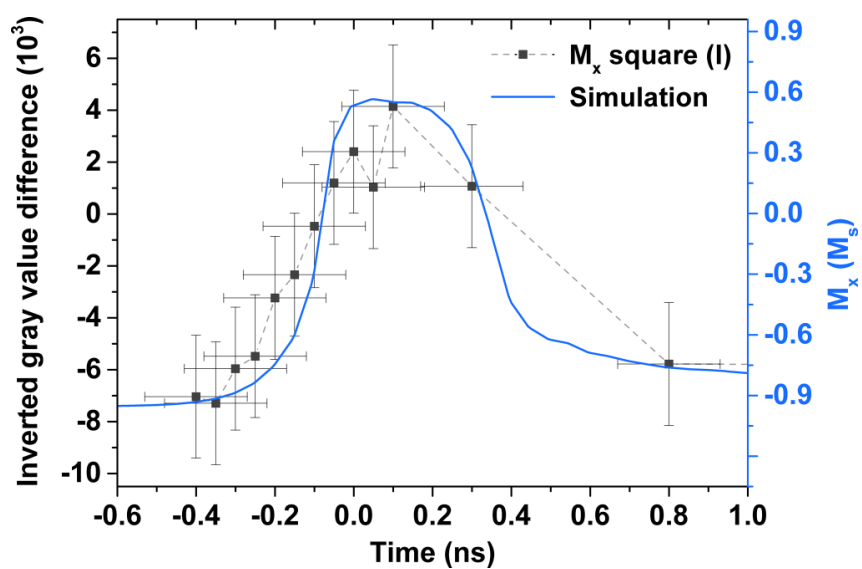

FIG. 7. (Color online) Spatially averaged inverse XMCD gray level of permalloy square I in Fig. 5 (black squares) with the averaged $M_{x}$ output of the micromagnetic simulation in Fig. 6 (blue solid curve). 


\section{CONCLUSION}

We have investigated the reaction of magnetic multidomain systems to intense subnanosecond Oersted excitation field pulses. The magnetization reorganization and formation of domains in permalloy microsquares forced into a homogeneously aligned magnetization configuration is studied with and without external magnetic fields. Initial Landau flux-closure structures featuring a central vortex core show unusually high core velocities exceeding $1 \mathrm{~km} / \mathrm{s}$ and a stable vortex chirality as well as polarity. This velocity exceeds the switching threshold of $\approx 300 \mathrm{~m} / \mathrm{s}$ and demonstrates the validity of the rigid particle model describing the vortex core motion on the curved eigenmode trajectory even at strong field amplitudes and high velocities.

In order to perform these experiments, we have built a new mobile microscopy endstation for the P04 beamline at PETRA III and used time-resolved full-field XMCD spectromicroscopy. This technique allows observing the domain pattern destruction and recovery with sub-100-nm spatial and subnanosecond temporal resolution. The presented data illustrate that high-resolution imaging is beneficial for these kinds of experiments because the spatially averaged magnetization signal alone does not include the whole magnetization dynamics. Moreover, the effects of impurities and inhomogeneities to the destruction and recovery dynamics can be studies with such a setup. The acquired movie sequences of the magnetization dynamics are supported by micromagnetic simulations reproducing the temporal shape of the magnetization decay and the transient spatial magnetization patterns.

\section{ACKNOWLEDGMENTS}

The authors would like to thank Pambos Charalambous (ZonePlates.com) for manufacturing of zone plate and grating condenser. Parts of this research were carried out at the light source PETRA III at DESY, a member of the Helmholtz Association (HGF). We would like to thank Leif Glaser, Jörn Seltmann, and Frank Scholz for assistance in using beamline P04. We thank Gennaro Abbati, Stefan Baumbach, and Johannes Overbuschmann for helping out during the beamtime shifts and the mechanical as well as electrical workshops of the University of Hamburg. Financial support is gratefully acknowledged from the Deutsche Forschungsgemeinschaft (DFG) through the Sonderforschungsbereich 668 (SFB 668) and the Hamburg Centre for Ultrafast Imaging (CUI). Additionally, we thank the Bundesministerium für Bildung und Forschung (BMBF) for funding through the Forschungsschwerpunkt 301 (05KS7GU4 and 05KS7UL1).
[1] T. Shinjo, T. Okuno, R. Hassdorf, K. Shigeto, and T. Ono, Science 289, 930 (2000).

[2] A. Wachowiak, J. Wiebe, M. Bode, O. Pietzsch, M. Morgenstern, and R. Wiesendanger, Science 298, 577 (2002).

[3] P. Fischer, M.-Y. Im, S. Kasai, K. Yamada, T. Ono, and A. Thiaville, Phys. Rev. B 83, 212402 (2011).

[4] S. Bohlens, B. Krüger, A. Drews, M. Bolte, G. Meier, and D. Pfannkuche, Appl. Phys. Lett. 93, 142508 (2008).

[5] S.-K. Kim, K.-S. Lee, Y.-S. Yu, and Y.-S. Choi, Appl. Phys. Lett. 92, 022509 (2008).

[6] M. Bolte, G. Meier, B. Krüger, A. Drews, R. Eiselt, L. Bocklage, S. Bohlens, T. Tyliszczak, A. Vansteenkiste, B. Van Waeyenberge, K. W. Chou, A. Puzic, and H. Stoll, Phys. Rev. Lett. 100, 176601 (2008).

[7] T. Kamionka, M. Martens, K. W. Chou, M. Curcic, A. Drews, G. Schütz, T. Tyliszczak, H. Stoll, B. Van Waeyenberge, and G. Meier, Phys. Rev. Lett. 105, 137204 (2010).

[8] S. Kasai, Y. Nakatani, K. Kobayashi, H. Kohno, and T. Ono, Phys. Rev. Lett. 97, 107204 (2006).

[9] V. S. Pribiag, I. N. Krivorotov, G. D. Fuchs, P. M. Braganca, O. Ozatay, J. C. Sankey, D. C. Ralph, and R. A. Buhrman, Nat. Phys. 3, 498 (2007).

[10] S.-B. Choe, Y. Acremann, A. Scholl, A. Bauer, A. Doran, J. Stöhr, and H. A. Padmore, Science 304, 420 (2004).

[11] B. Van Waeyenberge, A. Puzic, H. Stoll, K. W. Chou, T. Tyliszczak, R. Hertel, M. Fähnle, H. Brückl, K. Rott, G. Reiss, I. Neudecker, D. Weiss, C. H. Back, and G. Schütz, Nature (London) 444, 461 (2006).

[12] A. Vogel, T. Kamionka, M. Martens, A. Drews, K. W. Chou, T. Tyliszczak, H. Stoll, B. Van Waeyenberge, and G. Meier, Phys. Rev. Lett. 106, 137201 (2011).
[13] S. E. Stevenson, C. Moutafis, G. Heldt, R. V. Chopdekar, C. Quitmann, L. J. Heyderman, and J. Raabe, Phys. Rev. B 87, 054423 (2013)

[14] K. Y. Guslienko, X. F. Han, D. J. Keavney, R. Divan, and S. D. Bader, Phys. Rev. Lett. 96, 067205 (2006).

[15] H. Jung, Y.-S. Yu, K.-S. Lee, M.-Y. Im, P. Fischer, L. Bocklage, A. Vogel, M. Bolte, G. Meier, and S.-K. Kim, Appl. Phys. Lett. 97, 222502 (2010)

[16] B. Krüger, A. Drews, M. Bolte, U. Merkt, D. Pfannkuche, and G. Meier, Phys. Rev. B 76, 224426 (2007).

[17] M. Weigand, B. Van Waeyenberge, A. Vansteenkiste, M. Curcic, V. Sackmann, H. Stoll, T. Tyliszczak, K. Kaznatcheev, D. Bertwistle, G. Woltersdorf, C. H. Back, and G. Schütz, Phys. Rev. Lett. 102, 077201 (2009).

[18] K. Yamada, S. Kasai, Y. Nakatani, K. Kobayashi, H. Kohno, A. Thiaville, and T. Ono, Nat. Mater. 6, 270 (2007).

[19] K. Y. Guslienko, K.-S. Lee, and S.-K. Kim, Phys. Rev. Lett. 100, 027203 (2008).

[20] K.-S. Lee, S.-K. Kim, Y.-S. Yu, Y.-S. Choi, K. Y. Guslienko, H. Jung, and P. Fischer, Phys. Rev. Lett. 101, 267206 (2008).

[21] A. Vansteenkiste, K. W. Chou, M. Weigand, M. Curcic, V. Sackmann, H. Stoll, T. Tyliszczak, G. Woltersdorf, C. H. Back, G. Schütz, and B. Van Waeyenberge, Nat. Phys. 5, 332 (2009).

[22] J. Viefhaus, F. Scholz, S. Deinert, L. Glaser, M. Ilchen, J. Seltmann, P. Walter, and F. Siewert, Nucl. Instrum. Methods Phys. Res., Sect. A 710, 151 (2013).

[23] G. Schütz, W. Wagner, W. Wilhelm, P. Kienle, R. Zeller, R. Frahm, and G. Materlik, Phys. Rev. Lett. 58, 737 (1987).

[24] M. Schlie, Ph.D. thesis, University of Hamburg, 2013. 
[25] P. Wessels, M. Schlie, M. Wieland, J. Ewald, G. Abbati, S. Baumbach, J. Overbuschmann, T. Nisius, A. Vogel, A. Neumann, A. Meents, J. Viefhaus, H. P. Oepen, G. Meier, T. Wilhein, and M. Drescher, J. Phys.: Conf. Ser. 463, 012023 (2013).

[26] P. Wessels, J. Ewald, M. Wieland, T. Nisius, G. Abbati, S. Baumbach, J. Overbuschmann, A. Vogel, A. Neumann, J. Viefhaus, H. P. Oepen, G. Meier, T. Wilhein, and M. Drescher, J. Phys.: Conf. Ser. 499, 012009 (2014).

[27] P. Wessels, Ph.D. thesis, University of Hamburg, 2014.

[28] U. Vogt, M. Lindblom, P. Charalambous, B. Kaulich, and T. Wilhein, Opt. Lett. 31, 1465 (2006).

[29] P. Höß and K. Fleder, Proc. SPIE 4183, 127 (2001).

[30] See Supplemental Material at http://link.aps.org/supplemental/ 10.1103/PhysRevB.90.184417 for a video of the image sequence in Fig. 2.

[31] L. F. Yin, D. H. Wei, N. Lei, L. H. Zhou, C. S. Tian, G. S. Dong, X. F. Jin, L. P. Guo, Q. J. Jia, and R. Q. Wu, Phys. Rev. Lett. 97, 067203 (2006).

[32] T. Fischbacher, M. Franchin, G. Bordignon, and H. Fangohr, IEEE Trans. Magn. 43, 2896 (2007).
[33] H. Fangohr, T. Fischbacher, M. Franchin, G. Bordignon, J. Generowicz, A. Knittel, M. Walter, and M. Albert, NMAG User Manual Documentation, Release 0.2.1, University of Southampton, Highfield, Southampton SO17 1BJ, United Kingdom, 2012.

[34] A. Vogel, S. Wintz, T. Gerhardt, L. Bocklage, T. Strache, M.-Y. Im, P. Fischer, J. Fassbender, J. McCord, and G. Meier, Appl. Phys. Lett. 98, 202501 (2011).

[35] A. A. Thiele, Phys. Rev. Lett. 30, 230 (1973).

[36] M.-Y. Im, P. Fischer, K. Yamada, T. Sato, S. Kasai, Y. Nakatani, and T. Ono, Nat. Commun. 3, 983 (2012).

[37] See Supplemental Material at http://link.aps.org/supplemental/ 10.1103/PhysRevB.90.184417 for characteristic frames of the spatially resolved output of the NMAG simulation and at http://link.aps.org/supplemental/10.1103/PhysRevB.90.184417 for a video of this image sequence.

[38] See Supplemental Material at http://link.aps.org/supplemental/ 10.1103/PhysRevB.90.184417 for a video of the image sequence in Fig. 5.

[39] See Supplemental Material at http://link.aps.org/supplemental/ 10.1103/PhysRevB.90.184417 for a video of the image sequence in Fig. 6. 